\title{
Global Exponential Stability of Positive Almost Periodic Solutions for a Fishing Model with a Time-Varying Delay
}

\author{
Hong Zhang, ${ }^{1}$ Shuhua Gong, ${ }^{2}$ and Jianying Shao ${ }^{2}$ \\ ${ }^{1}$ College of Mathematics and Computer Science, Hunan University of Arts and Science, Changde, Hunan 415000, China \\ ${ }^{2}$ College of Mathematics, Physics and Information Engineering, Jiaxing University, Jiaxing, Zhejiang 314001, China \\ Correspondence should be addressed to Jianying Shao; shaojianying2008@aliyun.com
}

Received 18 October 2013; Revised 3 February 2014; Accepted 17 February 2014; Published 28 April 2014

Academic Editor: Shengqiang Liu

Copyright (c) 2014 Hong Zhang et al. This is an open access article distributed under the Creative Commons Attribution License, which permits unrestricted use, distribution, and reproduction in any medium, provided the original work is properly cited.

This paper is concerned with a nonautonomous fishing model with a time-varying delay. Under proper conditions, we employ a novel argument to establish a criterion on the global exponential stability of positive almost periodic solutions of the model with almost periodic coefficients and delays. Moreover, an example and its numerical simulation are given to illustrate the main results.

\section{Introduction}

In the classic study of population dynamics, the differential equation

$$
x^{\prime}=[L(t, x)-M(t, x)] x-F(t) x
$$

is widely used in fisheries [1-4], where $x=x(t)$ denotes the population biomass, $L(t, x)$ denotes the per-capita fecundity rate, $M(t, x)$ denotes the per-capita mortality rate, and $F(t)$ is the harvesting rate per-capita.

Let $L(t, x)$ be a Hills type function $[1,3]$

$$
L(t, x)=\frac{a}{1+(x / K)^{\gamma}}
$$

Denote $b(t)=M(t, x)+F(t)$. Taking account of the delay and the varying environments, Berezansky and Idels [5] proposed the following time-lag model based on (1):

$$
x^{\prime}(t)=x(t)\left[\frac{r(t)}{1+(x(t-\tau(t)) / K(t))^{\gamma}}-b(t)\right],
$$

where $r, b, K, \tau: R \rightarrow(0,+\infty)$ are almost periodic functions and parameter $\gamma>0$. Consequently, some theorems on the stability and existence of periodic solutions for (3) were established in Berezansky and Idels [5] and Wang [6]. For more details, we refer to the article of Wang [6].
In the real-world phenomena, the two foundations for the theory of nature selection are periodically and almost periodically varying environment. And the almost periodic effects are more frequent than the periodic effects (see $[7,8])$. Therefore, the effects of the almost periodic environment on the evolutionary theory have been extensively studied by a large number of researchers and some of these results can be found in [9-12]. For the reason of seasonal variation, it is not necessary to let (3) be exactly periodic but almost periodic instead. The problem of finding the global stability conditions for the positive almost periodic solution of (3) with almost periodic coefficients and delays becomes important. As pointed out in $[7,8]$, significant differences often appear in almost periodic problem by comparison with the periodic case. For example, contrary to periodic functions, there exists an almost periodic function $x(t)$ such that $x(t)>0$ for all $t \in R$ and $\inf _{t \in R} x(t)=0$. Hence, it is difficult to establish the existence, uniqueness, and global exponential stability of positive almost periodic solutions of (3). Moreover, to the best of our knowledge, there is no literature considering the global exponential stability of positive almost periodic solutions problem for (3) and its generalized equations.

Motivated by the above discussions, in this paper, a new approach will be developed to obtain a condition for the global exponential stability of the positive almost periodic solutions of (3), and the exponential convergent rate can be unveiled. 
For convenience, we introduce some notations. Given a bounded continuous function $g$ defined on $R$, let $g^{+}$and $g^{-}$ be defined as

$$
g^{+}=\sup _{t \in R} g(t), \quad g^{-}=\inf _{t \in R} g(t) .
$$

It will be assumed that

$$
\begin{gathered}
K^{-}>0, \quad b^{-}>0, \quad \tau^{+}>0, \\
r(t)>b(t) \quad \forall t \in R, \\
\left(\int_{t-\tau(t)}^{t}(r(s)-b(s)) d s\right)^{+}<+\infty, \\
\kappa=\left(\int_{t-\tau(t)}^{t} b(s) d s\right)^{+}<+\infty, \\
\times(t)\left(\frac{r(t)}{b(t)}-1\right)^{1 / \gamma} \\
\left.\times \exp \left\{\inf _{t \in R}^{t} \int_{t-\tau(t)}^{t}\left(\frac{r(s)}{1+(M / K(s))^{\gamma}}-b(s)\right) d s\right\}\right) \\
M=\left(K(t)\left(\frac{r(t)}{b(t)}-1\right)^{1 / \gamma}\right. \\
\quad \times \exp \left\{\sup _{t \in R}^{t} \int_{t-\tau(t)}^{(r(s)-b(s)) d s\})^{+}>\kappa .}\right.
\end{gathered}
$$

Then,

$$
\begin{gathered}
M \geq K(t)\left(\frac{r(t)}{b(t)}-1\right)^{1 / \gamma} \exp \left\{\sup _{t \in R} \int_{t-\tau(t)}^{t}(r(s)-b(s)) d s\right\} \\
\left(\frac{M}{K(t)}\right)^{\gamma} \geq\left(\frac{r(t)}{b(t)}-1\right) \exp \left\{\gamma \sup _{t \in R} \int_{t-\tau(t)}^{t}(r(s)-b(s)) d s\right\} \\
>\frac{r(t)}{b(t)}-1, \\
\left(\frac{r(t)}{1+(M / K(t))^{\gamma}}-b(t)\right)^{-} \leq \frac{r(t)}{1+(M / K(t))^{\gamma}}-b(t)<0
\end{gathered}
$$

$\forall t \in R$.

Let $R_{+}$denote nonnegative real number space, let $C=$ $C\left(\left[-\tau^{+}, 0\right], R\right)$ be the continuous functions space equipped with the usual supremum norm $\|\cdot\|$, and let $C_{+}=$ $C\left(\left[-\tau^{+}, 0\right], R_{+}\right)$. If $x(t)$ is defined on $\left[-\tau^{+}+t_{0}, \sigma\right)$ with $t_{0}, \sigma \in$ $R$, then we define $x_{t} \in C$ where $x_{t}(\theta)=x(t+\theta)$ for all $\theta \in\left[-\tau^{+}, 0\right]$.

Due to the biological interpretation of model (3), only positive solutions are meaningful and therefore admissible. Thus, we just consider admissible initial conditions

$$
x_{t_{0}}=\varphi, \quad \varphi \in C_{+}, \quad \varphi(0)>0 .
$$

We denote $x_{t}\left(t_{0}, \varphi\right)\left(x\left(t ; t_{0}, \varphi\right)\right)$ for an admissible solution of the admissible initial value problems (3) and (9). Also, let $\left[t_{0}, \eta(\varphi)\right)$ be the maximal right-interval of existence of $x_{t}\left(t_{0}, \varphi\right)$.

\section{Preliminary Results}

In this section, some lemmas and definitions will be presented, which are of importance in proving our main results in Section 3.

Definition 1 (see $[7,8]$ ). Let $u(t): R \rightarrow R$ be continuous in $t$. $u(t)$ is said to be almost periodic on $R$ if, for any $\varepsilon>0$, the set $T(u, \varepsilon)=\{\delta:|u(t+\delta)-u(t)|<\varepsilon$ for all $t \in R\}$ is relatively dense; that is, for any $\varepsilon>0$, it is possible to find a real number $l=l(\varepsilon)>0$ with the property that, for any interval with length $l(\varepsilon)$, there exists a number $\delta=\delta(\varepsilon)$ in this interval such that $\|u(t+\delta)-u(t)\|<\varepsilon$ for all $t \in R$.

From the theory of almost periodic functions in $[7,8]$, it follows that, for any $\epsilon>0$, it is possible to find a real number $l=l(\epsilon)>0$, and,for any interval with length $l(\epsilon)$, there exists a number $\delta=\delta(\epsilon)$ in this interval such that

$$
\begin{gathered}
|b(t+\delta)-b(t)|<\epsilon, \\
|r(t+\delta)-r(t)|<\epsilon, \\
|K(t+\delta)-K(t)|<\epsilon, \\
|\tau(t+\delta)-\tau(t)|<\epsilon,
\end{gathered}
$$

for all $t \in R$.

From (5) and Lemma 1.1 in Berezansky and Idels [5], we obtain the following lemma.

Lemma 2. Every solution $x\left(t ; t_{0}, \varphi\right)$ of (3) and (9) is persistent on $\left[t_{0}, \eta(\varphi)\right)$, and $\eta(\varphi)=+\infty$; that is, there exist two positive constants $K_{1}$ and $K_{2}$ such that

$$
K_{1} \leq \liminf _{t \rightarrow+\infty} x\left(t ; t_{0}, \varphi\right) \leq \limsup _{t \rightarrow+\infty} x\left(t ; t_{0}, \varphi\right) \leq K_{2} .
$$

Lemma 3. For every solution $x\left(t ; t_{0}, \varphi\right)$ of (3) and (9), there exists $t_{\varphi}>t_{0}$ such that

$$
\kappa<x\left(t ; t_{0}, \varphi\right)<M \quad \forall t \geq t_{\varphi} .
$$

Proof. Let $x(t)=x\left(t ; t_{0}, \varphi\right)$. It follows from Lemma 2 that

$$
0<\liminf _{t \rightarrow+\infty} x(t) \leq \limsup _{t \rightarrow+\infty} x(t)<+\infty
$$

We now give two cases to prove that there exists $T_{1}>t_{0}+\tau^{+}$ such that

$$
x(t) \leq M \quad \forall t \geq T_{1} .
$$

Case i. Suppose that

$$
x^{\prime}(t)<0, \quad \forall t \geq t_{0}+\tau^{+} .
$$


Then,

$$
L=\limsup _{t \rightarrow+\infty} x(t)=\lim _{t \rightarrow+\infty} x(t)=\lim _{t \rightarrow+\infty} x(t-\tau(t)) .
$$

By the fluctuation lemma [13, Lemma A.1], there exists a sequence $\left\{t_{p}\right\}_{p \geq 1}$ such that

$$
\begin{array}{r}
t_{p} \longrightarrow+\infty, \quad x\left(t_{p}\right) \longrightarrow \limsup _{t \rightarrow+\infty} x(t), \\
x^{\prime}\left(t_{p}\right) \longrightarrow 0 \quad \text { as } p \longrightarrow+\infty .
\end{array}
$$

Since $\left\{x_{t_{p}}\right\}$ is bounded and equicontinuous, by the AscoliArzela theorem, there exists a subsequence, still denoted by itself for simplicity of notation, such that

$$
x_{t_{p}} \longrightarrow \bar{\varphi} \quad \text { for some } \bar{\varphi} \in C_{+} \text {, as } p \longrightarrow+\infty \text {. }
$$

From (16), we get

$$
\bar{\varphi}(0)=L=\bar{\varphi}(\theta) \quad \forall \theta \in\left[-\tau^{+}, 0\right) .
$$

By the boundedness of the coefficients and delays, there is a subsequence of $\left\{t_{p}\right\}$, still denoted by $\left\{t_{p}\right\}$, such that $r\left(t_{p}\right)$, $b\left(t_{p}\right), K\left(t_{p}\right)$, and $\tau\left(t_{p}\right)$ are convergent to $\bar{r}, \bar{b}, \widehat{K}$, and $\bar{\tau}$, respectively. In view of the facts $L=\bar{\varphi}(-\bar{\tau})$ and $-\bar{\tau} \in\left[-\tau^{+}, 0\right]$, it follows from

$$
x^{\prime}\left(t_{p}\right)=x\left(t_{p}\right)\left[\frac{r\left(t_{p}\right)}{1+\left(x\left(t_{p}-\tau\left(t_{p}\right)\right) / K\left(t_{p}\right)\right)^{\gamma}}-b\left(t_{p}\right)\right]
$$

that (taking limits)

$$
\begin{aligned}
0 & =L\left[\frac{\bar{r}}{1+(\bar{\varphi}(-\bar{\tau}) / \widehat{K})^{\gamma}}-\bar{b}\right] \\
& =L\left[\frac{\bar{r}}{1+(L / \widehat{K})^{\gamma}}-\bar{b}\right],
\end{aligned}
$$

which yields

$$
L=\widehat{K}\left(\frac{\bar{r}}{\bar{b}}-1\right)^{1 / \gamma} \leq\left(K(t)\left(\frac{r(t)}{b(t)}-1\right)^{1 / \gamma}\right)^{+}<M .
$$

This implies that (14) holds.

Case ii. If there exists $\rho \geq t_{0}+\tau^{+}$such that $x^{\prime}(\rho) \geq 0$, then (3) yields

$$
\begin{array}{r}
0 \leq x^{\prime}(\rho)=x(\rho)\left[\frac{r(\rho)}{1+(x(\rho-\tau(\rho)) / K(\rho))^{\gamma}}-b(\rho)\right], \\
\quad \text { or } x(\rho-\tau(\rho)) \leq K(\rho)\left(\frac{r(\rho)}{b(\rho)}-1\right)^{1 / \gamma},
\end{array}
$$

and we have

$$
x^{\prime}(t) \leq x(t)[r(t)-b(t)], \quad \forall t \geq t_{0} .
$$

Integrating (24) from $\rho-\tau(\rho)$ to $\rho$, in view of (23), we obtain

$$
\begin{aligned}
x(\rho) \leq & x(\rho-\tau(\rho)) \exp \left\{\int_{\rho-\tau(\rho)}^{\rho}(r(s)-b(s)) d s\right\} \\
\leq & K(\rho)\left(\frac{r(\rho)}{b(\rho)}-1\right)^{1 / \gamma} \\
& \times \exp \left\{\sup _{t \in R} \int_{t-\tau(t)}^{t}(r(s)-b(s)) d s\right\} \\
\leq & M .
\end{aligned}
$$

For any $t>\rho$, with the same approach as that in derivation of (25), we can show

$$
x(t) \leq M \quad \text { if } x^{\prime}(t) \geq 0 .
$$

On the other hand, if $x^{\prime}(t)<0$ and $t>\rho$, we can choose $\rho \leq \bar{t}<t$ such that

$$
x^{\prime}(\bar{t})=0, \quad x^{\prime}(s)<0 \quad \forall s(\bar{t}, t],
$$

which, together with (26), yields

$$
x(t)<x(\bar{t}) \leq M .
$$

Thus, there must exist $T_{1}>\rho$ such that (14) holds.

Again from the fluctuation lemma [13, Lemma A.1], there exists a sequence $\left\{t_{q}\right\}_{q \geq 1}$ such that

$$
\begin{array}{r}
t_{q} \longrightarrow+\infty, \quad x\left(t_{q}\right) \longrightarrow \liminf _{t \rightarrow+\infty} x(t), \\
x^{\prime}\left(t_{q}\right) \longrightarrow 0 \text { as } q \longrightarrow+\infty .
\end{array}
$$

Since $\left\{x_{t_{t}}\right\}$ is bounded and equicontinuous, by the AscoliArzelá theorem, there exists a subsequence, still denoted by itself for simplicity of notation, such that

$$
x_{t_{q}} \longrightarrow \varphi^{*} \text { for some } \varphi^{*} \in C_{+} \text {, as } q \longrightarrow+\infty \text {. }
$$

We next divide our proof in two steps to show that there exists $T_{2}>\max \left\{T_{1}+\tau^{+}, t_{0}+\tau^{+}\right\}$such that

$$
x(t) \geq \kappa \quad \forall t \geq T_{2} .
$$

First, assume that

$$
x^{\prime}(t)>0, \quad \forall t \geq \max \left\{T_{1}+\tau^{+}, t_{0}+\tau^{+}\right\} .
$$

Then,

$$
0<A=\liminf _{t \rightarrow+\infty} x(t)=\lim _{t \rightarrow+\infty} x(t)=\lim _{t \rightarrow+\infty} x(t-\tau(t)) .
$$

According to (30) and (33), we get

$$
\varphi^{*}(0)=A=\varphi^{*}(\theta) \quad \forall \theta \in\left[-\tau^{+}, 0\right) .
$$


Without loss of generality, we assume that all $r\left(t_{q}\right), b\left(t_{q}\right)$, $K\left(t_{q}\right)$, and $\tau\left(t_{q}\right)$ are convergent to $r^{*}, b^{*}, K^{*}$, and $\tau^{*}$, respectively. This can be achieved because of almost periodicity. In view of (3) and (30), it follows from

$$
x^{\prime}\left(t_{q}\right)=x\left(t_{q}\right)\left[\frac{r\left(t_{q}\right)}{1+\left(x\left(t_{q}-\tau\left(t_{q}\right)\right) / K\left(t_{q}\right)\right)^{\gamma}}-b\left(t_{q}\right)\right]
$$

that (taking limits)

$$
\begin{aligned}
0 & =A\left[\frac{r^{*}}{1+\left(\varphi^{*}\left(-\tau^{*}\right) / K^{*}\right)^{\gamma}}-b^{*}\right] \\
& =A\left[\frac{r^{*}}{1+\left(A / K^{*}\right)^{\gamma}}-b^{*}\right],
\end{aligned}
$$

which, together with (8), yields

$$
\begin{aligned}
A= & K^{*}\left(\frac{r^{*}}{b^{*}}-1\right)^{1 / \gamma} \geq\left(K(t)\left(\frac{r(t)}{b(t)}-1\right)^{1 / \gamma}\right)^{-} \\
> & \left(K(t)\left(\frac{r(t)}{b(t)}-1\right)^{1 / \gamma}\right. \\
& \left.\quad \times \exp \left\{\inf _{t \in R} \int_{t-\tau(t)}^{t}\left(\frac{r(s)}{1+(M / K(s))^{\gamma}}-b(s)\right) d s\right\}\right)^{-} \\
= & \kappa .
\end{aligned}
$$

This implies that (31) holds.

Second, there exists $\rho^{*} \geq \max \left\{T_{1}+\tau^{+}, t_{0}+\tau^{+}\right\}$such that

$$
x^{\prime}\left(\rho^{*}\right) \leq 0 .
$$

This, together with (3), yields

$$
\begin{array}{r}
0 \geq x\left(\rho^{*}\right)\left[\frac{r\left(\rho^{*}\right)}{1+\left(x\left(\rho^{*}-\tau\left(\rho^{*}\right)\right) / K\left(\rho^{*}\right)\right)^{\gamma}}-b\left(\rho^{*}\right)\right], \\
\quad \text { or } x\left(\rho^{*}-\tau\left(\rho^{*}\right)\right) \geq K\left(\rho^{*}\right)\left(\frac{r\left(\rho^{*}\right)}{b\left(\rho^{*}\right)}-1\right)^{1 / \gamma},
\end{array}
$$

and we have

$$
\begin{aligned}
x^{\prime}(t) & =x(t)\left[\frac{r(t)}{1+(x(t-\tau(t)) / K(t))^{\gamma}}-b(t)\right] \\
& \geq x(t)\left[\frac{r(t)}{1+(M / K(t))^{\gamma}}-b(t)\right],
\end{aligned}
$$

for all $t \geq \max \left\{T_{1}+\tau^{+}, t_{0}+\tau^{+}\right\}$. Integrating (40) from $\rho^{*}-$ $\tau\left(\rho^{*}\right)$ to $\rho^{*}$, in view of $(6)$, we obtain

$$
\begin{aligned}
x\left(\rho^{*}\right) \geq & x\left(\rho^{*}-\tau\left(\rho^{*}\right)\right) \\
& \times \exp \left\{\int_{\rho^{*}-\tau\left(\rho^{*}\right)}^{\rho^{*}}\left(\frac{r(s)}{1+(M / K(s))^{\gamma}}-b(s)\right) d s\right\} \\
\geq & K\left(\rho^{*}\right)\left(\frac{r\left(\rho^{*}\right)}{b\left(\rho^{*}\right)}-1\right)^{1 / \gamma} \\
& \times \exp \left\{\inf _{t \in R} \int_{t-\tau(t)}^{t}\left(\frac{r(s)}{1+(M / K(s))^{\gamma}}-b(s)\right) d s\right\} \\
\geq & \kappa .
\end{aligned}
$$

For any $t>\rho^{*}$, with the same approach as that in derivation of (41), we can show

$$
x(t) \geq \kappa \quad \text { if } x^{\prime}(t) \leq 0 .
$$

On the other hand, if $x^{\prime}(t)>0$ and $t>\rho^{*}$, we can choose $\rho^{*} \leq \hat{t}<t$ such that

$$
x^{\prime}(\widehat{t})=0, \quad x^{\prime}(s)>0 \quad \forall s \in(\widehat{t}, t],
$$

which, together with (42), yields

$$
x(t)>x(\widehat{t}) \geq \kappa .
$$

Thus, there must exist $T_{2}>\rho^{*} \geq \max \left\{T_{1}+\tau^{+}, t_{0}+\tau^{+}\right\}$such that (31) holds.

In summary, (14) and (31) imply that there exists $t_{\varphi}>$ $\max \left\{T_{1}, T_{2}\right\}$ such that (12) holds. This ends the proof of Lemma 3.

Lemma 4. Let

$$
\begin{aligned}
\sup _{t \in R}\{- & {\left[b(t)+r(t) \kappa \frac{1}{K(t)} f_{m}\right] } \\
& +r(t) M \frac{1}{K(t)} f_{M} \tau(t) \\
& \times\left[b^{+}+r^{+} M \frac{1}{K^{-}} f_{M}+\frac{r^{+}}{1+\left(\kappa / K^{+}\right)^{\gamma}}\right] \\
& \left.+\frac{r(t)}{1+(\kappa / K(t))^{\gamma}}\right\}<0,
\end{aligned}
$$

where $f_{m}=\min _{x \in\left[\kappa / K^{+}, M / K^{-}\right]} f(x), \quad f_{M}=$ $\max _{x \in\left[\kappa / K^{+}, M / K^{-}\right]} f(x)$, and $f(x)=\gamma x^{\gamma-1} /\left(1+x^{\gamma}\right)^{2}$. Moreover, assume that $x(t)=x\left(t ; t_{0}, \varphi\right)$ is a solution of (3) with initial condition (9) and $\varphi^{\prime}$ is bounded continuous on $\left[-\tau^{+}, 0\right]$. Then, for any $\epsilon>0$, there exists $l=l(\epsilon)>0$, such that every interval $[\alpha, \alpha+l]$ contains at least one number $\delta$ for which there exists $N>0$ satisfying

$$
|x(t+\delta)-x(t)| \leq \epsilon, \quad \forall t>N .
$$


Proof. Define a continuous function $\Gamma(\mu)$ by setting

$$
\begin{aligned}
\Gamma(\mu)=\sup _{t \in R}\left\{-\left[b(t)+r(t) \kappa \frac{1}{K(t)} f_{m}-\mu\right]\right. \\
+r(t) M \frac{1}{K(t)} f_{M} \tau(t) \\
\quad \times\left[b^{+} e^{\mu \tau^{+}}+r^{+} M \frac{1}{K^{-}} f_{M} e^{2 \mu \tau^{+}}\right. \\
\left.+\frac{r^{+}}{1+\left(\kappa / K^{+}\right)^{\gamma}} e^{\mu \tau^{+}}\right] \\
\left.+\frac{r(t)}{1+(\kappa / K(t))^{\gamma}}\right\}, \quad \mu \in[0,1] .
\end{aligned}
$$

Then, we have

$$
\begin{aligned}
\Gamma(0)=\sup _{t \in R}\{- & {\left[b(t)+r(t) \kappa \frac{1}{K(t)} f_{m}\right] } \\
& +r(t) M \frac{1}{K(t)} f_{M} \tau(t) \\
& \times\left[b^{+}+r^{+} M \frac{1}{K^{-}} f_{M}+\frac{r^{+}}{1+\left(\kappa / K^{+}\right)^{\gamma}}\right] \\
& \left.+\frac{r(t)}{1+(\kappa / K(t))^{\gamma}}\right\}<0,
\end{aligned}
$$

which implies that there exist two constants $\eta>0$ and $\lambda \epsilon$ $(0,1]$ such that

$$
\begin{aligned}
& \Gamma(\lambda)=\sup _{t \in R}\left\{-\left[b(t)+r(t) \kappa \frac{1}{K(t)} f_{m}-\lambda\right]\right. \\
&+ r(t) M \frac{1}{K(t)} f_{M} \tau(t) \\
& \times\left[b^{+} e^{\lambda \tau^{+}}+r^{+} M\left(1 / K^{-}\right) f_{M} e^{2 \lambda \tau^{+}}\right. \\
&\left.+\frac{r^{+}}{1+\left(\kappa / K^{+}\right)^{\gamma}} e^{\lambda \tau^{+}}\right] \\
&\left.+\frac{r(t)}{1+(\kappa / K(t))^{\gamma}}\right\}<-\eta<0 .
\end{aligned}
$$

For $t \in\left(-\infty, t_{0}-\tau^{+}\right]$, we add the definition of $x(t)$ with $x(t) \equiv x\left(t_{0}-\tau^{+}\right)$. Set

$$
\begin{aligned}
\epsilon(\delta, t)= & -[b(t+\delta)-b(t)] x(t+\delta) \\
& +[r(t+\delta)-r(t)] \\
& \times \frac{x(t+\delta)}{1+(x(t+\delta-\tau(t+\delta)) / K(t+\delta))^{\gamma}}
\end{aligned}
$$

$$
\begin{aligned}
&+r(t)[ \frac{x(t+\delta)}{1+(x(t+\delta-\tau(t+\delta)) / K(t+\delta))^{\gamma}} \\
&\left.-\frac{x(t+\delta)}{1+(x(t+\delta-\tau(t+\delta)) / K(t))^{\gamma}}\right] \\
&+r(t)\left[\frac{x(t+\delta)}{1+(x(t+\delta-\tau(t+\delta)) / K(t))^{\gamma}}\right. \\
&\left.-\frac{x(t+\delta)}{1+(x(t+\delta-\tau(t)) / K(t))^{\gamma}}\right], \quad t \in R .
\end{aligned}
$$

By Lemma 3, the solution $x(t)$ is bounded and

$$
\kappa \leq x(t) \leq M, \quad \forall t \geq t_{\varphi}
$$

which implies that the right side of (3) is also bounded, and $x^{\prime}(t)$ is a bounded function on $\left[t_{0}-\tau^{+},+\infty\right)$. Thus, in view of the fact that $x(t) \equiv x\left(t_{0}-\tau^{+}\right)$for $t \in\left(-\infty, t_{0}-\tau^{+}\right]$, we obtain that $x(t)$ is uniformly continuous on $R$. From (10), for any $\epsilon>0$, there exists $l=l(\epsilon)>0$, such that every interval $[\alpha, \alpha+l], \alpha \in R$, contains a $\delta$ for which

$$
|\epsilon(\delta, t)| \leq \frac{1}{2} \frac{\eta \epsilon}{1+\tau^{+}}, \quad \forall t \in R .
$$

Let $N_{0} \geq \max \left\{t_{0}, t_{0}-\delta, t_{\varphi}+r, t_{\varphi}+r-\delta\right\}$. For $t \in R$, denote $u(t)=x(t+\delta)-x(t)$. Then, for all $t \geq N_{0}$, we get

$$
\begin{aligned}
& \frac{d u(t)}{d t}=-b(t)[x(t+\delta)-x(t)] \\
& +r(t) x(t+\delta) \\
& \times\left[\frac{1}{1+(x(t+\delta-\tau(t)) / K(t))^{\gamma}}\right. \\
& \left.-\frac{1}{1+(x(t-\tau(t)) / K(t))^{\gamma}}\right] \\
& +r(t) \frac{1}{1+(x(t-\tau(t)) / K(t))^{\gamma}} \\
& \times[x(t+\delta)-x(t)]+\epsilon(\delta, t) \\
& =-b(t) u(t)-r(t) x(t+\delta) \\
& \times \frac{1}{K(t)} f(\bar{\theta}(t)) u(t-\tau(t)) \\
& +\frac{r(t)}{1+(x(t-\tau(t)) / K(t))^{\gamma}} u(t)+\epsilon(\delta, t) \\
& =-[b(t)+r(t) x(t+\delta) \\
& \left.\times \frac{1}{K(t)} f(\bar{\theta}(t))\right] u(t)+r(t) x(t+\delta) \\
& \times \frac{1}{K(t)} f(\bar{\theta}(t)) \int_{t-\tau(t)}^{t} u^{\prime}(s) d s \\
& +\frac{r(t)}{1+(x(t-\tau(t)) / K(t))^{\gamma}} u(t)+\epsilon(\delta, t)
\end{aligned}
$$




$$
\begin{gathered}
=-\left[b(t)+r(t) x(t+\delta) \frac{1}{K(t)} f(\bar{\theta}(t))\right] u(t) \\
+r(t) x(t+\delta) \frac{1}{K(t)} f(\bar{\theta}(t)) \\
\times \int_{t-\tau(t)}^{t}[-b(s) u(s)-r(s) x(s+\delta) \\
\quad \times \frac{1}{K(s)} f(\bar{\theta}(s)) u(s-\tau(s)) \\
\quad+\frac{r(s)}{1+(x(s-\tau(s)) / K(s))^{\gamma}} u(s) \\
+\epsilon(\delta, s)] d s \\
+\frac{r(t)}{1+(x(t-\tau(t)) / K(t))^{\gamma}} u(t) \\
+\epsilon(\delta, t), \quad
\end{gathered}
$$

where $\bar{\theta}(t)$ lies between $x(t+\delta-\tau(t)) / K(t)$ and $x(t-$ $\tau(t)) / K(t)$.

Calculating the upper right derivative of $e^{\lambda t}|u(t)|$ yields

$$
\begin{aligned}
& D^{-}\left(e^{\lambda t}|u(t)|\right) \\
& \leq \lambda e^{\lambda t}|u(t)| \\
& +e^{\lambda t}\left\{-\left[b(t)+r(t) x(t+\delta) \frac{1}{K(t)} f(\bar{\theta}(t))\right]|u(t)|\right. \\
& +r(t) x(t+\delta) \frac{1}{K(t)} f(\bar{\theta}(t)) \\
& \times \int_{t-\tau(t)}^{t} \mid-b(s) u(s) \\
& -r(s) x(s+\delta) \frac{1}{K(s)} f(\bar{\theta}(s)) \\
& \times u(s-\tau(s)) \\
& +\frac{r(s)}{1+(x(s-\tau(s)) / K(s))^{\gamma}} \\
& \times u(s)+\epsilon(\delta, s) \mid d s \\
& \left.+\frac{r(t)}{1+(x(t-\tau(t)) / K(t))^{\gamma}}|u(t)|+|\epsilon(\delta, t)|\right\} \\
& \leq-\left[b(t)+r(t) \kappa \frac{1}{K(t)} f_{m}-\lambda\right] e^{\lambda t}|u(t)| \\
& +r(t) M \frac{1}{K(t)} f_{M}
\end{aligned}
$$

$$
\begin{aligned}
& \times \int_{t-\tau(t)}^{t}[ b^{+} e^{\lambda(t-s)} e^{\lambda s}|u(s)| \\
&+r^{+} M \frac{1}{K^{-}} f_{M} e^{\lambda(t-s+\tau(s))} e^{\lambda(s-\tau(s))} \\
& \times|u(s-\tau(s))| \\
&\left.+\frac{r^{+}}{1+\left(\kappa / K^{+}\right)^{\gamma}} e^{\lambda(t-s)} e^{\lambda s}|u(s)|\right] d s \\
&+e^{\lambda t} \tau^{+} \frac{1}{2} \frac{\eta \epsilon}{1+\tau^{+}}+\frac{r(t)}{1+(\kappa / K(t))^{\gamma}}|u(t)| e^{\lambda t} \\
&+e^{\lambda t} \frac{1}{2} \frac{\eta \epsilon}{1+\tau^{+}}, \quad \forall t \geq N_{0} .
\end{aligned}
$$

Let

$$
U(t)=\sup _{-\infty<s \leq t}\left\{e^{\lambda s}|u(s)|\right\}
$$

It is obvious that $e^{\lambda t}|u(t)| \leq U(t)$ and $U(t)$ is nondecreasing. Now, we distinguish two cases to finish the proof.

Case One. Consider

$$
U(t)>e^{\lambda t}|u(t)| \quad \forall t \geq N_{0}
$$

We claim that

$$
U(t) \equiv U\left(N_{0}\right) \quad \text { is a constant } \forall t \geq N_{0} .
$$

Assume, by way of contradiction, that (57) does not hold. Then, there exists $t_{1}>N_{0}$ such that $U\left(t_{1}\right)>U\left(N_{0}\right)$. Since

$$
e^{\lambda t}|u(t)| \leq U\left(N_{0}\right) \quad \forall t \leq N_{0} .
$$

There must exist $\beta \in\left(N_{0}, t_{1}\right)$ such that

$$
e^{\lambda \beta}|u(\beta)|=U\left(t_{1}\right) \geq U(\beta),
$$

which contradicts (56). This contradiction implies that (57) holds. It follows that there exists $t_{2}>N_{0}$ such that

$$
|u(t)| \leq e^{-\lambda t} U(t)=e^{-\lambda t} U\left(N_{0}\right)<\epsilon \quad \forall t \geq t_{2} .
$$

Case Two. There is a $t_{0}^{*} \geq N_{0}$ such that $U\left(t_{0}^{*}\right)=e^{\lambda t_{0}^{*}}\left|u\left(t_{0}^{*}\right)\right|$. Then, in view of (49), (52), and (54), we get

$$
\begin{aligned}
0 \leq & \left.D^{-}\left(e^{\lambda t}|u(t)|\right)\right|_{t=t_{0}^{*}} \\
\leq & -\left[b\left(t_{0}^{*}\right)+r\left(t_{0}^{*}\right) \kappa \frac{1}{K\left(t_{0}^{*}\right)} f_{m}-\lambda\right] e^{\lambda t_{0}^{*}}\left|u\left(t_{0}^{*}\right)\right| \\
& +r\left(t_{0}^{*}\right) M \frac{1}{K\left(t_{0}^{*}\right)} f_{M}
\end{aligned}
$$




$$
\begin{aligned}
& \times \int_{t_{0}^{*}-\tau\left(t_{0}^{*}\right)}^{t_{0}^{*}}\left[b^{+} e^{\lambda\left(t_{0}^{*}-s\right)} e^{\lambda s}|u(s)|\right. \\
& +r^{+} M \frac{1}{K^{-}} f_{M} e^{\lambda\left(t_{0}^{*}-s+\tau(s)\right)} e^{\lambda(s-\tau(s))} \\
& \times|u(s-\tau(s))| \\
& \left.+\frac{r^{+}}{1+\left(\kappa / K^{+}\right)^{\gamma}} e^{\lambda\left(t_{0}^{*}-s\right)} e^{\lambda s}|u(s)|\right] d s \\
& +\frac{r\left(t_{0}^{*}\right)}{1+\left(\kappa / K\left(t_{0}^{*}\right)\right)^{\gamma}}\left|u\left(t_{0}^{*}\right)\right| e^{\lambda t_{0}^{*}}+e^{\lambda t_{0}^{*}} \frac{1}{2} \eta \epsilon \\
& \leq\left\{-\left[b\left(t_{0}^{*}\right)+r\left(t_{0}^{*}\right) \kappa \frac{1}{K\left(t_{0}^{*}\right)} f_{m}-\lambda\right]\right. \\
& +r\left(t_{0}^{*}\right) M \frac{1}{K\left(t_{0}^{*}\right)} f_{M} \tau\left(t_{0}^{*}\right) \\
& \times\left[b^{+} e^{\lambda \tau^{+}}+r^{+} M \frac{1}{K^{-}} f_{M} e^{2 \lambda \tau^{+}}\right. \\
& \left.\left.+\frac{r^{+}}{1+\left(\kappa / K^{+}\right)^{\gamma}} e^{\lambda \tau^{+}}\right]+\frac{r\left(t_{0}^{*}\right)}{1+\left(\kappa / K\left(t_{0}^{*}\right)\right)^{\gamma}}\right\} U\left(t_{0}^{*}\right) \\
& +e^{\lambda t_{0}^{*}} \frac{1}{2} \eta \epsilon \\
& \leq-\eta U\left(t_{0}^{*}\right)+e^{\lambda t_{0}^{*}} \eta \epsilon,
\end{aligned}
$$

which yields that

$$
e^{\lambda t_{0}^{*}}\left|u\left(t_{0}^{*}\right)\right|=U\left(t_{0}^{*}\right)<\epsilon e^{\lambda t_{0}^{*}}, \quad\left|u\left(t_{0}^{*}\right)\right|<\epsilon .
$$

For any $t>t_{0}^{*}$, with the same approach as that in derivation of (62), we can show

$$
e^{\lambda t}|u(t)|<\epsilon e^{\lambda t}, \quad|u(t)|<\epsilon,
$$

if $U(t)=e^{\lambda t}|u(t)|$.

On the other hand, if $U(t)>e^{\lambda t}|u(t)|$ and $t>t_{0}^{*}$, we can choose $t_{0}^{*} \leq t_{3}<t$ such that

$$
U\left(t_{3}\right)=e^{\lambda t_{3}}\left|u\left(t_{3}\right)\right|, \quad U(s)>e^{\lambda s}|u(s)| \quad \forall s \in\left(t_{3}, t\right],
$$

which, together with (63), yields

$$
\left|u\left(t_{3}\right)\right|<\epsilon .
$$

With a similar argument as that in the proof of Case One, we can show that

$$
U(s) \equiv U\left(t_{3}\right) \quad \text { is a constant } \forall s \in\left(t_{3}, t\right],
$$

which implies that

$$
|u(t)|<e^{-\lambda t} U(t)=e^{-\lambda t} U\left(t_{3}\right)=\left|u\left(t_{3}\right)\right| e^{-\lambda\left(t-t_{3}\right)}<\epsilon .
$$

In summary, there must exist $N>\max \left\{t_{0}^{*}, N_{0}, t_{2}\right\}$ such that $|u(t)| \leq \epsilon$ holds for all $t>N$. The proof of Lemma 4 is now complete.

\section{Main Results}

In this section, we establish sufficient conditions on the existence and global exponential stability of almost periodic solutions of (3).

Theorem 5. Under the assumptions of Lemma 4, (3) has at least one positive almost periodic solution $x^{*}(t)$. Moreover, $x^{*}(t)$ is globally exponentially stable; that is, there exist constants $K_{\varphi, x^{*}}$ and $t_{\varphi, x^{*}}$ such that

$$
\left|x\left(t ; t_{0}, \varphi\right)-x^{*}(t)\right|<K_{\varphi, x^{*}} e^{-\lambda t} \quad \forall t>t_{\varphi, x^{*}} .
$$

Proof. Let $v(t)=v\left(t ; t_{0}, \varphi^{v}\right)$ be a solution of (3) with initial conditions satisfying the assumptions in Lemma 4 . We also add the definition of $v(t)$ with $v(t) \equiv v\left(t_{0}-\tau^{+}\right)$for all $t \in$ $\left(-\infty, t_{0}-\tau^{+}\right]$. Set

$$
\begin{aligned}
& \epsilon(k, t)=-\left[b\left(t+t_{k}\right)-b(t)\right] v\left(t+t_{k}\right) \\
&+\left[r\left(t+t_{k}\right)-r(t)\right] \\
& \times \frac{v\left(t+t_{k}\right)}{1+\left(v\left(t+t_{k}-\tau\left(t+t_{k}\right)\right) / K\left(t+t_{k}\right)\right)^{\gamma}} \\
&++\frac{x(t)\left[\frac{v\left(t+t_{k}\right)}{1+\left(v\left(t+t_{k}-\tau\left(t+t_{k}\right)\right) / K\left(t+t_{k}\right)\right)^{\gamma}}\right.}{} \\
&\left.-\frac{v\left(t+t_{k}\right)}{1+\left(x\left(t+t_{k}-\tau\left(t+t_{k}\right)\right) / K(t)\right)^{\gamma}}\right] \\
&+r(t)\left[\frac{v\left(t+t_{k}\right)}{1+\left(v\left(t+t_{k}-\tau\left(t+t_{k}\right)\right) / K(t)\right)^{\gamma}}\right. \\
&-\frac{\left.1\left(t+t_{k}-\tau(t)\right) / K(t)\right)^{\gamma}}{1+(v(t)} t \in R,
\end{aligned}
$$

where $\left\{t_{k}\right\}$ is any sequence of real numbers. By Lemma 3, the solution $v(t)$ is bounded and

$$
\kappa<v(t)<M, \quad \forall t \geq t_{\varphi^{v}}
$$

which implies that the right side of (3) is also bounded, and $v^{\prime}(t)$ is a bounded function on $\left[t_{0}-r,+\infty\right)$. Thus, in view of the fact that $v(t) \equiv v\left(t_{0}-r\right)$ for $t \in\left(-\infty, t_{0}-r\right]$, we obtain that $v(t)$ is uniformly continuous on $R$. Then, from the almost periodicity of $r, b, K$, and $\tau$, we can select a sequence $\left\{t_{k}\right\} \rightarrow$ $+\infty$ such that

$$
\begin{gathered}
\left|a\left(t+t_{k}\right)-a(t)\right| \leq \frac{1}{k}, \\
\left|b\left(t+t_{k}\right)-b(t)\right| \leq \frac{1}{k} \\
\left|\tau\left(t+t_{k}\right)-\tau(t)\right| \leq \frac{1}{k}, \quad|\epsilon(k, t)| \leq \frac{1}{k}, \quad \forall t .
\end{gathered}
$$

Since $\left\{v\left(t+t_{k}\right)\right\}_{k=1}^{+\infty}$ is uniformly bounded and equiuniformly continuous, by Arzala-Ascoli Lemma and diagonal 
selection principle, we can choose a subsequence $\left\{t_{k_{j}}\right\}$ of $\left\{t_{k}\right\}$, such that $v\left(t+t_{k_{j}}\right)$ (for convenience, we still denote it by $\left.v\left(t+t_{k}\right)\right)$ uniformly converges to a continuous function $x^{*}(t)$ on any compact set of $R$, and

$$
\kappa \leq x^{*}(t) \leq M, \quad \forall t \in R
$$

Now, we prove that $x^{*}(t)$ is a solution of (2). In fact, for any $t \geq t_{0}$ and $\Delta t \in R$, from (71), we have

$$
\begin{aligned}
& x^{*}(t+\Delta t)-x^{*}(t) \\
& =\lim _{k \rightarrow+\infty}\left[v\left(t+\Delta t+t_{k}\right)-v\left(t+t_{k}\right)\right] \\
& =\lim _{k \rightarrow+\infty} \int_{t}^{t+\Delta t}\left\{-b\left(\mu+t_{k}\right) v\left(\mu+t_{k}\right)+r\left(\mu+t_{k}\right)\right. \\
& \times\left(v\left(\mu+t_{k}\right)\right. \\
& \left.\left.\times\left(1+\left(\frac{v\left(\mu+t_{k}-\tau\left(\mu+t_{k}\right)\right)}{K\left(\mu+t_{k}\right)}\right)^{\gamma}\right)^{-1}\right)\right\} d \mu \\
& =\lim _{k \rightarrow+\infty} \int_{t}^{t+\Delta t}\left\{-b(\mu) v\left(\mu+t_{k}\right)+r(\mu)\right. \\
& \times \frac{v\left(\mu+t_{k}\right)}{1+\left(v\left(\mu+t_{k}-\tau(\mu)\right) / K(\mu)\right)^{\gamma}} \\
& +\epsilon(k, \mu)\} d \mu \\
& =\int_{t}^{t+\Delta t}\left\{x^{*}(\mu)\left[\frac{r(\mu)}{1+\left(x^{*}(\mu-\tau(\mu)) / K(\mu)\right)^{\gamma}}-b(\mu)\right]\right\} d \mu \\
& +\lim _{k \rightarrow+\infty} \int_{t}^{t+\Delta t} \epsilon(k, \mu) d \mu \\
& =\int_{t}^{t+\Delta t}\left\{x^{*}(\mu)\left[\frac{r(\mu)}{1+\left(x^{*}(\mu-\tau(\mu)) / K(\mu)\right)^{\gamma}}-b(\mu)\right]\right\} d \mu,
\end{aligned}
$$

where $t+\Delta t \geq t_{0}$. Consequently, (73) implies that

$$
\frac{d}{d t}\left\{x^{*}(t)\right\}=x^{*}(t)\left[\frac{r(t)}{1+\left(x^{*}(t-\tau(t)) / K(t)\right)^{\gamma}}-b(t)\right] \text {. }
$$

Therefore, $x^{*}(t)$ is a solution of (3).

Secondly, we prove that $x^{*}(t)$ is an almost periodic solution of (3). With the help of Lemma 4 , for any $\varepsilon>0$, there exists $l=l(\varepsilon)>0$, such that every interval $[\alpha, \alpha+l]$ contains at least one number $\delta$ for which there exists $N>0$ satisfying

$$
|v(t+\delta)-v(t)| \leq \varepsilon, \quad \forall t>N .
$$

Then, for any fixed $s \in R$, we can find a sufficient large positive integer $N_{1}>N$ such that, for any $k>N_{1}$,

$$
s+t_{k}>N, \quad\left|v\left(s+t_{k}+\delta\right)-v\left(s+t_{k}\right)\right| \leq \varepsilon .
$$

Let $k \rightarrow+\infty$; we obtain

$$
\left|x^{*}(s+\delta)-x^{*}(s)\right| \leq \varepsilon,
$$

which implies that $x^{*}(t)$ is an almost periodic solution of (3).

Finally, we prove that $x^{*}(t)$ is globally exponentially stable.

Let $x(t)=x\left(t ; t_{0}, \varphi\right)$ and $y(t)=x(t)-x^{*}(t)$, where $t \epsilon$ $\left[t_{0}-\tau^{+},+\infty\right)$. Then,

$$
\begin{aligned}
& y^{\prime}(t) \\
& =-b(t) y(t)+r(t) \\
& \times\left[\frac{x(t)}{1+(x(t-\tau(t)) / K(t))^{\gamma}}-\frac{x^{*}(t)}{1+\left(x^{*}(t-\tau(t)) / K(t)\right)^{\gamma}}\right] \\
& =-b(t) y(t)+r(t) x(t) \\
& \times\left[\frac{1}{1+(x(t-\tau(t)) / K(t))^{\gamma}}-\frac{1}{1+\left(x^{*}(t-\tau(t)) / K(t)\right)^{\gamma}}\right] \\
& +r(t) \frac{1}{1+\left(x^{*}(t-\tau(t)) / K(t)\right)^{\gamma}} y(t) \\
& =-b(t) y(t)-r(t) x(t) \frac{1}{K(t)} f(\widehat{\theta}(t)) y \\
& \times(t-\tau(t))+r(t) \frac{1}{1+\left(x^{*}(t-\tau(t)) / K(t)\right)^{\gamma}} y(t) \\
& =-\left[b(t)+r(t) x(t) \frac{1}{K(t)} f(\widehat{\theta}(t))\right] y(t) \\
& +r(t) x(t) \frac{1}{K(t)} f(\widehat{\theta}(t)) \int_{t-\tau(t)}^{t} y^{\prime}(s) d s \\
& +r(t) \frac{1}{1+\left(x^{*}(t-\tau(t)) / K(t)\right)^{\gamma}} y(t) \\
& =-\left[b(t)+r(t) x(t) \frac{1}{K(t)} f(\widehat{\theta}(t))\right] y(t) \\
& +r(t) x(t) \frac{1}{K(t)} f(\hat{\theta}(t)) \\
& \times \int_{t-\tau(t)}^{t}\left[-b(s) y(s)-r(s) x(s) \frac{1}{K(s)}\right. \\
& \times f(\widehat{\theta}(s)) y(s-\tau(s)) \\
& \left.+r(s) \frac{1}{1+\left(x^{*}(s-\tau(s)) / K(s)\right)^{\gamma}} y(s)\right] d s \\
& +r(t) \frac{1}{1+\left(x^{*}(t-\tau(t)) / K(t)\right)^{\gamma}} y(t),
\end{aligned}
$$

where $t \geq t_{0}+\tau^{+}$and $\widehat{\theta}(t)$ lies between $x(t-\tau(t)) / K(t)$ and $x^{*}(t-\tau(t)) / K(t)$. 
It follows from Lemma 3 that there exists $t_{\varphi, x^{*}}>t_{0}+\tau^{+}$ such that

$$
\begin{gathered}
\kappa \leq x(t), \quad x^{*}(t) \leq M, \\
\forall t \in\left[t_{\varphi, x^{*}}-\tau^{+},+\infty\right) .
\end{gathered}
$$

We consider the Lyapunov functional

$$
V(t)=|y(t)| e^{\lambda t} .
$$

Calculating the upper left derivative of $V(t)$ along the solution $y(t)$ of (78), we have

$$
\begin{aligned}
& D^{-}(V(t)) \\
& \leq \lambda|y(t)| e^{\lambda t} \\
& +e^{\lambda t}\left\{-\left[b(t)+r(t) x(t) \frac{1}{K(t)} f(\widehat{\theta}(t))\right]|y(t)|\right. \\
& +r(t) x(t) \frac{1}{K(t)} f(\widehat{\theta}(t)) \\
& \times \int_{t-\tau(t)}^{t} \mid-b(s) y(s)-r(s) x(s) \frac{1}{K(s)} \\
& \times f(\widehat{\theta}(s)) y(s-\tau(s))+r(s) \\
& \times \frac{1}{1+\left(x^{*}(s-\tau(s)) / K(s)\right)^{\gamma}} \\
& \times y(s) \mid d s \\
& +r(t) \frac{1}{1+\left(x^{*}(t-\tau(t)) / K(t)\right)^{\gamma}} \\
& \times|y(t)|\} \\
& \leq-\left[b(t)+r(t) \kappa \frac{1}{K(t)} f_{m}-\lambda\right] e^{\lambda t}|y(t)| \\
& +r(t) M \frac{1}{K(t)} f_{M} \int_{t-\tau(t)}^{t}\left[b^{+} e^{\lambda(t-s)} e^{\lambda s}|y(s)|\right. \\
& +r^{+} M \frac{1}{K^{-}} f_{M} e^{\lambda(t-s+\tau(s))} \\
& \times e^{\lambda(s-\tau(s))}|y(s-\tau(s))| \\
& +\frac{r^{+}}{1+\left(\kappa / K^{+}\right)^{\gamma}} e^{\lambda(t-s)} \\
& \left.\times e^{\lambda s}|y(s)|\right] d s \\
& +\frac{r(t)}{1+(\kappa / K(t))^{\gamma}}|y(t)| e^{\lambda t}, \quad \forall t>t_{\varphi, x^{*}} .
\end{aligned}
$$

We claim that

$$
V(t)=|y(t)| e^{\lambda t}
$$

$$
\begin{aligned}
& <e^{\lambda t_{\varphi, x^{*}}}\left(\max _{t \in\left[t_{0}-r, t_{\varphi, x^{*}}\right]}\left|x(t)-x^{*}(t)\right|+1\right) \\
& :=K_{\varphi, x^{*}} \quad \forall t>t_{\varphi, x^{*}} .
\end{aligned}
$$

Contrarily, there must exist $t_{*}>t_{\varphi, x^{*}}$ such that

$$
V\left(t_{*}\right)=K_{\varphi, x^{*}}, \quad V(t)<K_{\varphi, x^{*}} \quad \forall t \in\left[t_{0}-\tau^{+}, t_{*}\right) .
$$

Together with (55), (81), and (83), we obtain

$$
\begin{aligned}
& 0 \leq D^{-}\left(V\left(t_{*}\right)\right) \\
& \leq-\left[b\left(t_{*}\right)+r\left(t_{*}\right) \kappa \frac{1}{K\left(t_{*}\right)} f_{m}-\lambda\right] e^{\lambda t_{*}}\left|y\left(t_{*}\right)\right| \\
& +r\left(t_{*}\right) M \frac{1}{K\left(t_{*}\right)} f_{M} \\
& \times \int_{t_{*}-\tau\left(t_{*}\right)}^{t_{*}}\left[b^{+} e^{\lambda\left(t_{*}-s\right)} e^{\lambda s}|y(s)|\right. \\
& +r^{+} M \frac{1}{K^{-}} f_{M} e^{\lambda\left(t_{*}-s+\tau(s)\right)} \\
& \times e^{\lambda(s-\tau(s))}|y(s-\tau(s))| \\
& +\frac{r^{+}}{1+\left(\kappa / K^{+}\right)^{\gamma}} \\
& \left.\times e^{\lambda\left(t_{*}-s\right)} e^{\lambda s}|y(s)|\right] d s \\
& +\frac{r\left(t_{*}\right)}{1+\left(\kappa / K\left(t_{*}\right)\right)^{\gamma}}\left|y\left(t_{*}\right)\right| e^{\lambda t} \\
& \leq\left\{-\left[b\left(t_{*}\right)+r\left(t_{*}\right) \kappa \frac{1}{K\left(t_{*}\right)} f_{m}-\lambda\right]\right. \\
& +r\left(t_{*}\right) M \frac{1}{K\left(t_{*}\right)} f_{M} \tau\left(t_{*}\right) \\
& \times\left[b^{+} e^{\lambda \tau^{+}}+r^{+} M \frac{1}{K^{-}} f_{M} e^{2 \lambda \tau^{+}}\right. \\
& \left.\left.+\frac{r^{+}}{1+\left(\kappa / K^{+}\right)^{\gamma}} e^{\lambda \tau^{+}}\right]+\frac{r\left(t_{*}\right)}{1+\left(\kappa / K\left(t_{*}\right)\right)^{\gamma}}\right\} K_{\varphi, x^{*}} .
\end{aligned}
$$

Thus,

$$
\begin{aligned}
0 \leq & -\left[b\left(t_{*}\right)+r\left(t_{*}\right) \kappa \frac{1}{K\left(t_{*}\right)} f_{m}-\lambda\right] \\
& +r\left(t_{*}\right) M \frac{1}{K\left(t_{*}\right)} f_{M} \tau\left(t_{*}\right)
\end{aligned}
$$




$$
\begin{gathered}
\times\left[b^{+} e^{\lambda \tau^{+}}+r^{+} M \frac{1}{K^{-}} f_{M} e^{2 \lambda \tau^{+}}\right. \\
\left.+\frac{r^{+}}{1+\left(\kappa / K^{+}\right)^{\gamma}} e^{\lambda \tau^{+}}\right] \\
+\frac{r\left(t_{*}\right)}{1+\left(\kappa / K\left(t_{*}\right)\right)^{\gamma}}
\end{gathered}
$$

which contradicts (49). Hence, (82) holds. It follows that

$$
|y(t)|<K_{\varphi, x^{*}} e^{-\lambda t} \quad \forall t>t_{\varphi, x^{*}} .
$$

This completes the proof of Theorem 5.

\section{An Example}

In this section, we present an example to check the validity of the results we obtained in the previous sections.

Example 1. Consider the following fishing model with timevarying delay:

$$
\begin{aligned}
& x^{\prime}(t) \\
& \quad=x(t)\left[\frac{3+(1 / 20) \sin \sqrt{2} t}{1+\left(x\left(t-\cos ^{2} t / 40\right) / 4\right)^{2}}-1-\frac{1}{20} \sin \sqrt{3} t\right] .
\end{aligned}
$$

Obviously, $\gamma=2, r(t)=3+(1 / 20) \sin \sqrt{2} t, b(t)=$ $1+(1 / 20) \sin \sqrt{3} t, K(t)=4, \tau(t)=\cos ^{2} t / 40, f(x)=$ $\gamma x^{\gamma-1} /\left(1+x^{\gamma}\right)^{2}$. By calculating, we obtain

$$
\begin{gathered}
\left(\int_{t-\tau(t)}^{t}(r(s)-b(s)) d s\right)^{+} \approx 0.0525, \\
\left(\int_{t-\tau(t)}^{t} b(s) d s\right)^{+} \approx 0.02625, \\
\kappa \approx 5.3587, \\
M \approx 4 \sqrt{\frac{42}{19}} e^{21 / 400} \approx 6.2677>\kappa, \\
f_{m} \approx 0.2625, \quad f_{M} \approx 0.3430, \\
\sup _{t \in R}\left\{-\left[b(t)+r(t) \kappa \frac{1}{K(t)} f_{m}\right]\right. \\
+r(t) M \frac{1}{K(t)} f_{M} \tau(t) \\
\quad \times\left[b^{+}+r^{+} M \frac{1}{K^{-}} f_{M}+\frac{r^{+}}{1+\left(\kappa / K^{+}\right)^{\gamma}}\right] \\
\left.+\frac{r(t)}{1+(\kappa / K(t))^{\gamma}}\right\} \\
-0.8296<0,
\end{gathered}
$$

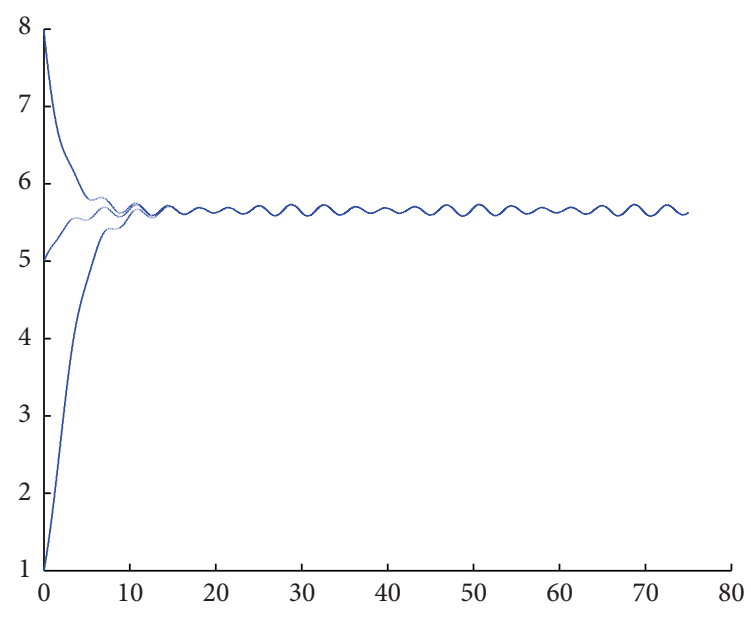

FIgURE 1: Numerical solution $x(t)$ of system (87) for initial value $\varphi(t) \equiv 1,5,8$.

which imply that (87) satisfies the assumptions of Theorem 5. Therefore, (87) has a unique positive almost periodic solution $x^{*}(t)$, which is globally exponentially stable with the exponential convergent rate $\lambda \approx 0.001$. The numerical simulation in Figure 1 strongly supports the conclusion.

Remark 6. Most recently, by using Mawhin continuation theorem, criteria ensuring the local existence of almost periodic solutions for the fishing model (3) are established in Li et al. [14], where the global exponential convergence for almost periodic solution is not touched. Unfortunately, as pointed out by Wang and Zhang [15] and Ortega [16], for the essential reason that the compact condition is not suitable for the almost periodic function family, the coincidence degree cannot be used to solve almost periodic problem. Hence, the mapping $N$ of Lemma3.3 in Li et al. [14] is not $L$ compact and the existence of almost periodic solutions for (3) cannot hold. Moreover, to the best of our knowledge, there is no research on the global exponential stability of positive almost periodic solutions to the fishing model (3). We also mention that all results in $[5,6,9,10,12]$ cannot be applied to imply that all solutions of (87) with initial values (9) converge exponentially to the positive almost periodic solution. Here, we employ a novel proof to establish some criteria to guarantee the existence and global exponential stability of positive almost periodic solutions for fishing model with time-varying coefficients and delays. This implies that the results of this paper are contributed to complement the previous references in this topic.

\section{Conflict of Interests}

The authors declare no conflict of interests. They also declare that they have no financial and personal relationships with other people or organizations that can inappropriately influence their work; there is no professional or other personal interest of any nature or kind in any product, service, and/or 
company that could be construed as influencing the position presented in, or the review of, this present paper.

\section{Acknowledgments}

The authors would like to express the sincere appreciation to the reviewers for their helpful comments in improving the presentation and quality of the paper. In particular, the authors express the sincere gratitude to Professor Bingwen Liu for the helpful discussion when this revision work is carried out. This work was supported by the National Natural Science Foundation of China (Grant no. 11201184), the Natural Scientific Research Fund of Zhejiang Provincial of China (Grant no. LY12A01018), and the Natural Scientific Research Fund of Zhejiang Provincial Education Department of China (Grant no. Z201122436).

\section{References}

[1] L. Berezansky, E. Braverman, and L. Idels, "Delay differential equations with Hill's type growth rate and linear harvesting," Computers \& Mathematics with Applications, vol. 49, no. 4, pp. 549-563, 2005.

[2] K. Gopalsamy, Stability and Oscillations in Delay Differential Equations of Population Dynamics, vol. 74 of Mathematics and Its Applications, Kluwer Academic, Dordrecht, The Netherlands, 1992.

[3] M. Kot, Elements of Mathematical Ecology, Cambridge University Press, Cambridge, UK, 2001.

[4] Y. Kuang, Delay Differential Equations with Applications in Population Dynamics, vol. 191 of Mathematics in Science and Engineering, Academic Press, Boston, Mass, USA, 1993.

[5] L. Berezansky and L. Idels, "Stability of a time-varying fishing model with delay," Applied Mathematics Letters. An International Journal of Rapid Publication, vol. 21, no. 5, pp. 447-452, 2008.

[6] X. Wang, "Stability and existence of periodic solutions for a time-varying fishing model with delay," Nonlinear Analysis: Real World Applications, vol. 11, no. 5, pp. 3309-3315, 2010.

[7] A. M. Fink, Almost Periodic Differential Equations, vol. 377 of Lecture Notes in Mathematics, Springer, Berlin, Germany, 1974.

[8] C. Y. He, Almost Periodic Differential Equation, Higher Education Publishing House, Beijing, China, 1992 (Chinese).

[9] J. Meng, "Global exponential stability of positive pseudo almost periodic solutions for a model of hematopoiesis," Abstract and Applied Analysis, vol. 2013, Article ID 463076, 7 pages, 2013.

[10] S. Ahmad and I. M. Stamova, "Almost necessary and sufficient conditions for survival of species," Nonlinear Analysis: Real World Applications, vol. 5, no. 1, pp. 219-229, 2004.

[11] T. Yoshizawa, Stability Theory and the Existence of Periodic Solutions and Almost Periodic Solutions, vol. 14, Springer, Berlin, Germany, 1975, Applied Mathematical Sciences.

[12] B. Liu, "New results on the positive almost periodic solutions for a model of hematopoiesis," Nonlinear Analysis: Real World Applications, vol. 17, pp. 252-264, 2014.

[13] H. L. Smith, An Introduction to Delay Differential Equations with Applications to the Life Sciences, vol. 57 of Texts in Applied Mathematics, Springer, New York, NY, USA, 2011.

[14] X. Li, Y. Li, and C. He, "Positive almost periodic solutions for a time-varying fishing model with delay," International Journal of Differential Equations, vol. 2011, Article ID 354016, 12 pages, 2011.

[15] X. Wang and H. Zhang, "A new approach to the existence, nonexistence and uniqueness of positive almost periodic solution for a model of hematopoiesis," Nonlinear Analysis: Real World Applications, vol. 11, no. 1, pp. 60-66, 2010.

[16] R. Ortega, "Degree theory and almost periodic problems", in Differential Equations, Chaos and Variational Problems, vol. 75 of Progress in Nonlinear Differential Equations and Their Applications, pp. 345-356, Springer, New York, NY, USA, 2008. 


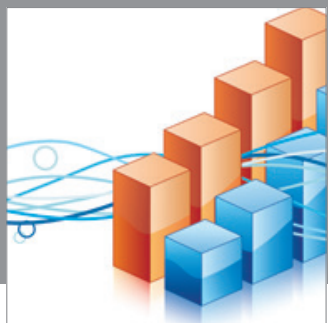

Advances in

Operations Research

mansans

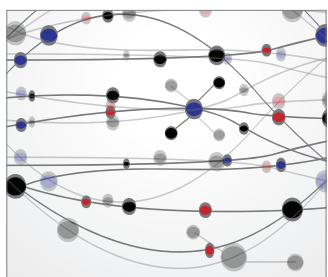

The Scientific World Journal
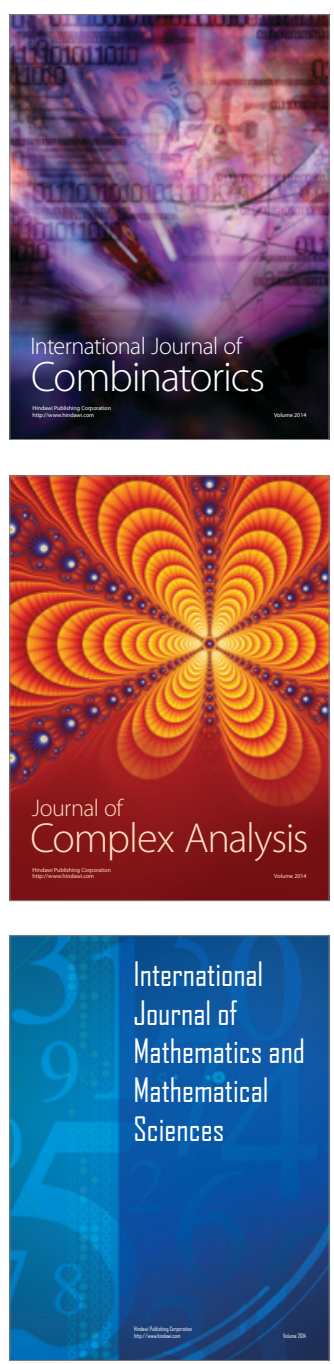
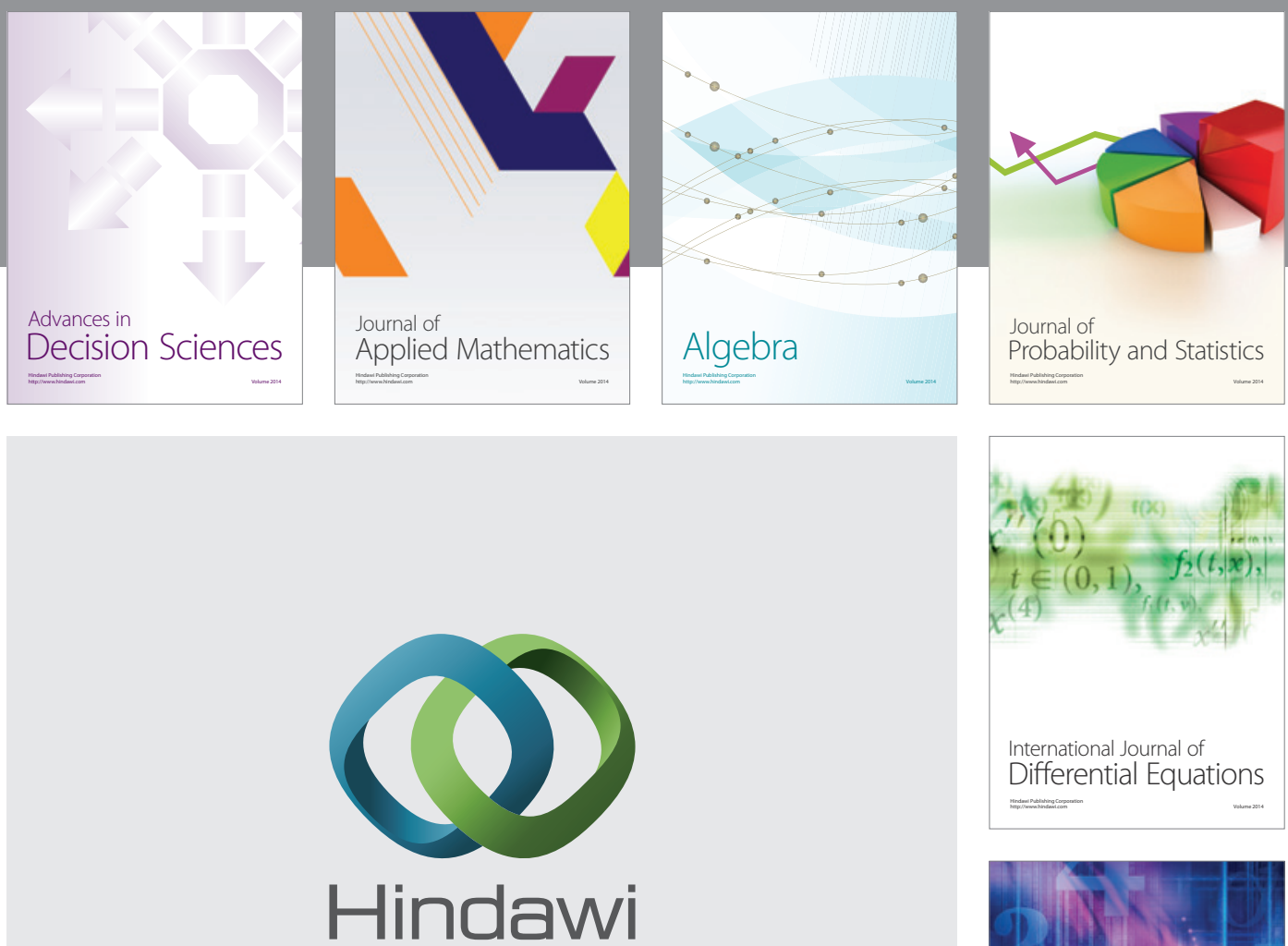

Submit your manuscripts at http://www.hindawi.com
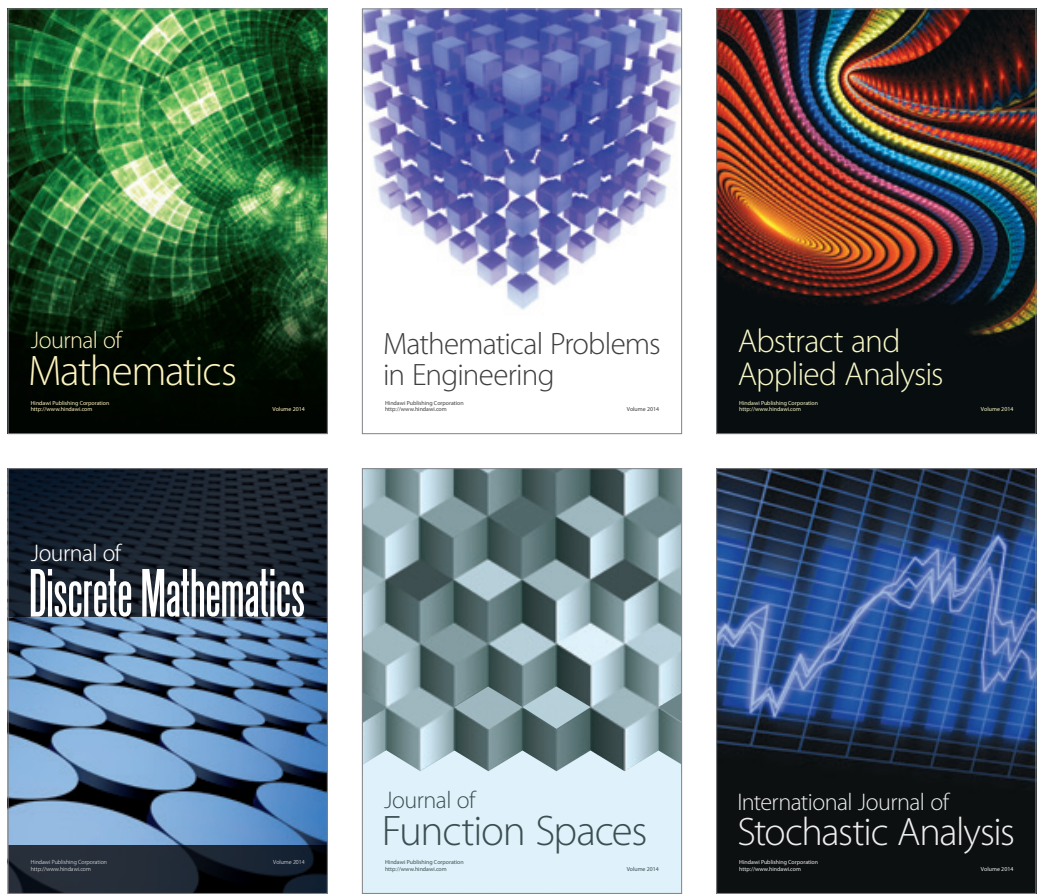

Journal of

Function Spaces

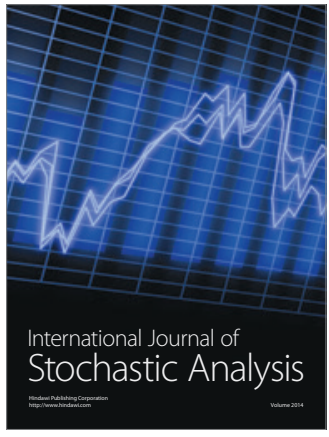

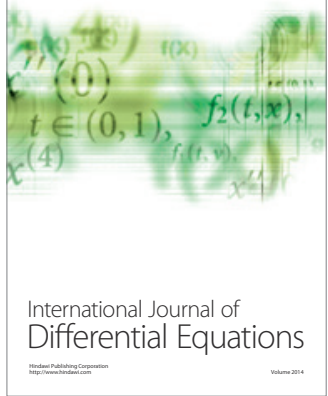
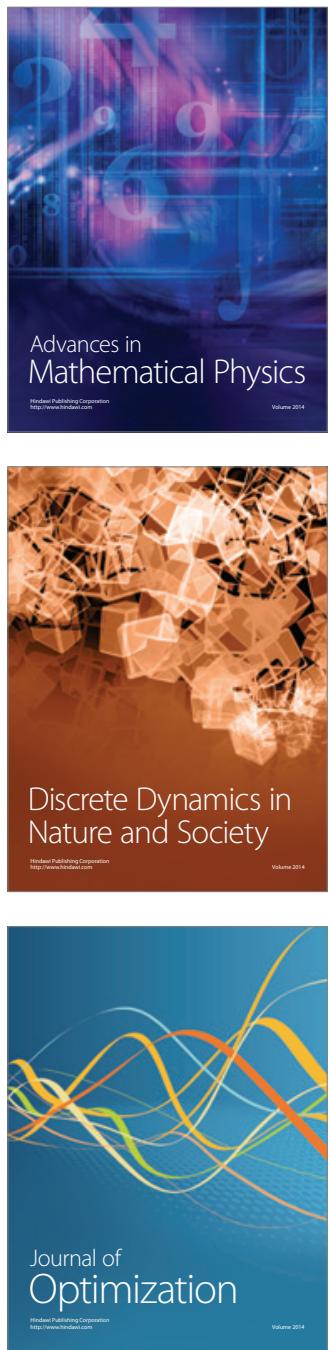